\title{
Operational immune tolerance towards transplanted allogeneic pancreatic islets in mice and a non-human primate
}

\author{
Midhat H. Abdulreda ${ }^{1,2,3,4}$. Dora M. Berman ${ }^{1,2}$. Alexander Shishido ${ }^{1} \cdot$ Christopher Martin $^{1} \cdot$ Maged Hossameldin $^{1}$. \\ Ashley Tschiggfrie ${ }^{1} \cdot$ Luis F. Hernandez $^{1}$ - Ana Hernandez ${ }^{1}$ - Camillo Ricordi ${ }^{1,2,5}$ • Jean-Marie Parel ${ }^{4}$. \\ Ewa Jankowska-Gan ${ }^{6}$. William J. Burlingham ${ }^{6}$ • Esdras A. Arrieta-Quintero ${ }^{4}$ - Victor L. Perez ${ }^{4,7}$ • Norma S. Kenyon ${ }^{1,2,3}$. \\ Per-Olof Berggren ${ }^{1,2,8}$
}

Received: 13 July 2018 / Accepted: 14 December 2018 / Published online: 31 January 2019

(C) Springer-Verlag GmbH Germany, part of Springer Nature 2019

\begin{abstract}
Aims/hypothesis Patients with autoimmune type 1 diabetes transplanted with pancreatic islets to their liver experience significant improvement in quality of life through better control of blood sugar and enhanced awareness of hypoglycaemia. However, longterm survival and efficacy of the intrahepatic islet transplant are limited owing to liver-specific complications, such as immediate blood-mediated immune reaction, hypoxia, a highly enzymatic and inflammatory environment and locally elevated levels of drugs including immunosuppressive agents, all of which are injurious to islets. This has spurred a search for new islet transplant sites and for innovative ways to achieve long-term graft survival and efficacy without life-long systemic immunosuppression and its complications.

Methods We used our previously established approach of islet transplant in the anterior chamber of the eye in allogeneic recipient mouse models and a baboon model of diabetes, which were treated transiently with anti-CD154/CD40L blocking antibody in the peri-transplant period. Survival of the intraocular islet allografts was assessed by direct visualisation in the eye and metabolic variables (blood glucose and C-peptide measurements). We evaluated longitudinally the cytokine profile in the local microenvironment of the intraocular islet allografts, represented in aqueous humour, under conditions of immune rejection vs tolerance. We also evaluated the recall response in the periphery of the baboon recipient using delayed-type hypersensitivity (DTH) assay, and in mice after repeat transplant in the kidney following initial transplant with allogeneic islets in the eye or kidney.

Results Results in mice showed $>300$ days immunosuppression-free survival of allogeneic islets transplanted in the eye or kidney. Notably, $>70 \%$ of tolerant mice, initially transplanted in the eye, exhibited $>400$ days of graft survival after retransplant in the kidney without immunosuppression compared with $\sim 30 \%$ in mice that were initially transplanted in the kidney. Cytokine and DTH data provided evidence of T helper 2-driven local and peripheral immune regulatory mechanisms in support of operational immune tolerance towards the islet allografts in both models.
\end{abstract}

Midhat H. Abdulreda and Dora M. Berman contributed equally.

Electronic supplementary material The online version of this article (https://doi.org/10.1007/s00125-019-4814-4) contains peer-reviewed but unedited supplementary material, which is available to authorised users.

Midhat H. Abdulreda

mabdulreda@miami.edu

Per-Olof Berggren

per-olof.berggren@ki.se

1 Diabetes Research Institute and Cell Transplant Center, University of Miami Miller School of Medicine, 1450 NW 10th Ave,

Miami, FL 33136, USA

2 Department of Surgery, University of Miami Miller School of Medicine, Miami, FL, USA

3 Department of Microbiology and Immunology, University of Miami Miller School of Medicine, Miami, FL, USA
4 Bascom Palmer Eye Institute, University of Miami Miller School of Medicine, Miami, FL, USA

5 Diabetes Research Institute Federation, Hollywood, FL, USA

6 Department of Surgery, School of Medicine and Public Health, University of Wisconsin, Madison, WI, USA

7 Present address: Duke Ophthalmology, Duke University, Durham, NC, USA

8 The Rolf Luft Research Center for Diabetes and Endocrinology, Karolinska Institutet, Karolinska University Hospital L1, SE-17176 Stockholm, Sweden 


\section{Research in context}

\section{What is already known about this subject?}

- Islet transplantation in the liver improves quality of life in individuals with type 1 diabetes

- Long-term graft efficacy of intrahepatic islet transplants is restricted owing to liver-specific limitations, despite continued immunosuppression

- New sites are critically needed where long-term survival and efficacy of transplanted islets can be achieved without chronic immunosuppression and its complications

\section{What is the key question?}

- Can long-term survival of allogeneic islets transplanted in the anterior chamber of the eye be achieved without continued immunosuppression?

\section{What are the new findings?}

- Allogeneic islets transplanted in the anterior chamber of the eye survived long-term without continued immunosuppression in murine and non-human primate (baboon) models of diabetes

- Long-term survival of intraocular islet allografts was supported by locally elevated immune regulatory cytokines in both the mice and the baboon

- Long-term survival of the allografts was also associated with operational immune tolerance in the periphery of the diabetic recipient mice and baboon

How might this impact on clinical practice in the foreseeable future?

- The current findings may have a significant impact on clinical islet transplant therapy through induction of immune tolerance towards transplanted allogeneic islets

Conclusions/interpretation We are currently evaluating the safety and efficacy of intraocular islet transplantation in a phase 1 clinical trial. In this study, we demonstrate immunosuppression-free long-term survival of intraocular islet allografts in mice and in a baboon using transient peri-transplant immune intervention. These results highlight the potential for inducing islet transplant immune tolerance through the intraocular route. Therefore, the current findings are conceptually significant and may impact markedly on clinical islet transplantation in the treatment of diabetes.

Keywords Allogeneic rejection - Anterior chamber of the eye Immune tolerance induction and maintenance . Immunosuppression-free · Intraocular transplantation · Long-term graft survival · Non-invasive longitudinal intravital imaging $\cdot$ Pancreatic islet transplant $\cdot$ Th2 cytokines

$\begin{array}{ll}\text { Abbreviations } \\ \text { DTH } & \text { Delayed-type hypersensitivity } \\ \text { IBMIR } & \text { Immediate blood-mediated immune reaction } \\ \text { IEQ } & \text { Islet equivalents } \\ \text { PBMC } & \text { Peripheral blood mononuclear cell } \\ \text { POD } & \text { Postoperative day } \\ \text { STZ } & \text { Streptozotocin } \\ \text { Th } & \text { T helper } \\ \text { TT/D } & \text { Tetanus toxoid and diphtheria }\end{array}$

\section{Introduction}

To restore or induce immune tolerance is the holy grail of organ, tissue and cell replacement therapies through transplantation. Current transplantations rely on immunosuppression to prevent immune-mediated graft rejection. Pancreatic islet transplantation is a promising therapy for autoimmune type 1 diabetes. A recent phase 3 trial by the Clinical Islet Transplantation Consortium on islet transplantation to the liver in individuals with uncontrolled type 1 diabetes showed significant improvement in blood sugar control and reduction in the number of episodes of hypoglycaemia [1]. While this and other previous reports have shown significant enhancement in the quality of life of the recipients [2], it has also become evident that the long-term benefits of intrahepatic islet transplantation are limited by liver-specific complications, such as low oxygen tension, immediate blood-mediated immune reaction (IBMIR), a highly enzymatic/inflammatory environment and elevated drug levels including immunosuppressive agents, all of which are injurious to intrahepatic islet grafts [3]. This has spurred a vigorous search for new islet 
transplant sites and several sites are being investigated, such as the omentum, the subcutaneous and intramuscular spaces, the bone marrow and the anterior chamber of the eye [4-9]. It should also be noted that realising the full potential of clinical islet transplantation as a long-lasting therapy in type 1 diabetes requires not only protection of the transplanted islets from immune damage but also protection from other 'non-immune' injury as has been shown to occur in the liver. Therefore, there is a keen interest in the transplantation field in finding innovative ways to induce transplant immune tolerance to ensure long-term graft acceptance (e.g. islets) without the complications of immunosuppression $[10,11]$.

We now present evidence that islet transplantation in the anterior chamber of the eye offers various unique benefits including the potential for long-term graft survival without sustained immunosuppression. Based on this and our extensive experience with intraocular islet transplantation [8, 12-14], we have become interested in the anterior chamber of the eye as a clinical site for islet transplantation and are currently evaluating its safety and efficacy in legally blind type 1 diabetes patients in a phase 1 clinical trial (ClinTrials.gov registration no. NCT02846571). We believe clinical islet transplantation in the eye is promising in the treatment of type 1 diabetes $[8,9]$. Our findings consistently indicate that islets thrive immediately after transplantation into the anterior chamber of the eye, likely due to the high local oxygen tension in the aqueous humour, which is comparable with that in the native pancreas [15-17]. Additionally, islets transplanted in the eye can be monitored non-invasively and longitudinally [8], which enables early detection and timely intervention against rejection if or when needed. Our previous studies have shown that intraocular islet grafts are retained indefinitely in syngeneic MHC-matched recipient mouse models of diabetes $[12,18]$ but they are rejected in allogeneic (i.e. MHC-mismatched) recipients when transplanted without immune intervention $[13,19]$. The current studies, however, demonstrate the feasibility of long-term immunosuppression-free survival of islet allografts in the eye of allogeneic diabetic recipient mice and a baboon treated transiently with immunotherapy. Importantly, the technical features of islet transplantation in the eye combined with evidence for associated induction of operational immune tolerance in the clinically relevant nonhuman primate model further highlight this technique's promise in clinical application.

\section{Methods}

Animals and reagents All studies were performed under protocols approved by the University of Miami's Institutional Animal Care and Use Committee (IACUC). The anti-CD154 antibody (mouse clone MR-1) was obtained from Bio-X-Cell (USA) and for non-human primates (clone 5C8) was obtained from NonHuman Primate Reagent Resource (AI126683 and OD10976) at the National Institutes of Health (NIH). See electronic supplementary material (ESM) Methods for further details.

Pancreatic islet isolation and islet transplantation Islet isolation from donor mice (DBA/2 both sexes) or a male non-sibling donor baboon and transplantation into the anterior chamber of the eye or under the kidney capsule of recipient mice $(\mathrm{C} 57 \mathrm{BL} / 6$; B6, both sexes) or the eye of the female baboon $(n=1)$, were performed as previously described in detail [8, 20-23] (also see ESM Methods for further details). The recipient female baboon (4 years old, $8.2 \mathrm{~kg}$ body weight at the time of transplant) was rendered diabetic by partial pancreatectomy 557 days prior to islet transplantation, followed by streptozotocin (STZ) treatment (see EMS Methods) and was infused on the day of transplantation with 40,000 islet equivalents (IEQs) (i.e. $4900 \mathrm{IEQ} / \mathrm{kg}$ body weight) in the right eye only. However, there was a technical complication, possibly due to known inter-individual variability in islet quality from preparation-to-preparation following isolation from non-human primate donors; this resulted in islet clumping during the first few days after transplantation. Islets that were not in direct contact with the iris after infusion into the anterior chamber 'clumped' together and disappeared within 10 days after transplant, as was confirmed by direct monitoring of the islet graft. Consequently, the remaining islet mass following this initial phase was estimated at $\sim 600 \mathrm{IEQ} / \mathrm{kg}$ based on the islet graft surface area before and after the clumping occurred and this was assumed to be the functional islet mass in the recipient baboon throughout the study. After diabetes induction, as well as post islet cell transplant, blood glucose plasma levels were monitored two or three times daily via heel stick using a OneTouch Ultra Glucometer (LifeScan, Milpitas, CA, USA). Subcutaneous insulin was administered (Humulin R; Eli Lilly, Indianapolis, IN, USA or Humulin R + Lantus; Sanofi-Aventis, Bridgewater, NJ, USA) as needed, based on an individualised sliding scale, aiming for fasting and postprandial plasma glucose levels of 9.00$15.00 \mathrm{mmol} / 1 \mathrm{post}-\mathrm{STZ}$ and prior to transplantation, and 6.00 $12.00 \mathrm{mmol} / \mathrm{l}$ after islet transplantation. Clinical monitoring was performed by daily observation and regular monitoring of clinical signs, fluid balance, body weight, body temperature and nutritional intake. Blood samples were drawn pre- and posttransplant to assess fasting plasma C-peptide (enhanced chemiluminescence immunoassay, Cobas 6000 analyzer; Roche Diagnostic, USA), serum chemistries, cell blood count (CBC), $\mathrm{HbA}_{1 \mathrm{c}}$ (DCA 2000+ Analyzer; Bayer, Elkhart, IN, USA) and cytomegalovirus (CMV) levels (not shown) were measured as previously described in detail [24].

Trans vivo delayed-type hypersensitivity assay Trans vivo delayed-type hypersensitivity (DTH) assay was performed as previously described in detail $[25,26]$ to assess immune reactivity (or lack thereof) of the recipient baboon to the specific islet donor (see EMS Methods for further details). The extent of bystander immune suppression was measured as \% 
inhibition of recall antigen response in trans vivo DTH in the presence of donor antigen according to the following formula:

$$
\begin{aligned}
\text { \%Inhibition }= & 1-[(\text { Recall Ag }+ \text { Test Ag }) /(\text { Recall Ag })] \\
& \times 100 \%
\end{aligned}
$$

where the 'Recall Ag' is tetanus toxoid and diphtheria (TT/D) antigen and the 'Test $\mathrm{Ag}$ ' is soluble test antigen prepared from frozen splenocytes $\left(12 \times 10^{6}\right.$ in $\left.100 \mu \mathrm{l}\right)$ by sonication followed by centrifugation $(16,000 g)$ to remove large cell fragments [26]. The splenocytes were obtained from the donor baboon from which the transplanted islets were isolated (i.e. 'Donor Ag') and third-party control baboons (i.e. Ctrl Ag 1 and $\mathrm{Ctrl} \mathrm{Ag}$ 2). The following antibodies were used for cytokine neutralisation in the trans vivo DTH assay: anti-human IL-10 LEAF (used at $10 \mu \mathrm{g}$; BioLegend 501407, Clone: JES3-9D7); anti-human L-12/IL-35 p35 (1 $\mu \mathrm{g}$; R\&D Systems MAB1570); anti-human Ebi3 (1 $\mu \mathrm{g}$; a generous gift from D. Vignali); and anti-human TGF- $\beta 1$ ( $25 \mu \mathrm{g}$; R\&D Systems AB-100-NA). The following Ig isotype controls were used: mouse IgG1 $(1 \mu \mathrm{g})$; rabbit $\operatorname{IgG}(25 \mu \mathrm{g})$ and rat IgG1 K

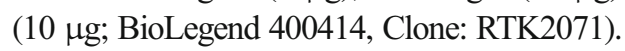

Statistical analysis Experimenters were blinded to group assignment and outcome assessment whenever possible. Data were plotted and analysed in GraphPad Prism version 6.07. Statistical analyses were done using parametric and nonparametric comparisons tests (unpaired Student's $t$ test and one-way ANOVA followed by Tukey's multiple comparison test) and, where applicable, data were fit with linear or nonlinear regression functions. Islet allograft survival analysis was based on Kaplan-Meier survival curves and comparison of the median survival times was done by the Logrank (MantelCox) test. Frequency distribution histograms were generated using automatic binning and the histograms were fit with non-linear Gaussian function; correlation analysis was done using the non-parametric Spearman's correlation coefficient in Prism. Asterisks indicate significance with $p$ value $\leq 0.05$.

\section{Results}

Long-term survival of islet allografts following transplantation in the eye or kidney of mice in the absence of immunosuppression We transplanted full MHC-mismatched allogeneic $\mathrm{DBA} / 2\left(\mathrm{H}-2^{\mathrm{d}}\right)$ donor islets into the eye anterior chamber or under the kidney subcapsular space of STZ-induced diabetic C57BL/6 $\left(\mathrm{B} 6 ; \mathrm{H}-2^{\mathrm{b}}\right)$ recipient mice. The recipients were treated transiently with anti-CD154 (CD40L) antibody (20-30 mg/kg; clone MR-1 or isotype Ig control or PBS) in the peri-transplantation period (day -3 and -1 ), on the day of transplantation (day 0 ) and on postoperative days (POD) 3 and 7. We assessed the survival of the intraocular islet allografts before and after stopping immunosuppression by direct examination of the intraocular islet grafts using non-invasive intravital imaging as previously described [13] (Fig. 1a, b), and by longitudinal monitoring of blood glucose of the recipients (Fig. 1c). The results showed normalisation of blood glucose following islet transplantation into the anterior chamber of one eye or in the kidney of diabetic recipient mice. Recipients of islets in either site maintained normal blood sugar levels (mean non-fasting blood glucose $\leq 11.11 \mathrm{mmol} / \mathrm{l}$ ) when treated with the anti-CD154 antibody MR-1, whereas those treated with Ig control returned to hyperglycaemia (blood glucose $>16.66 \mathrm{mmol} / \mathrm{l}$ ) (Fig. 1c). Notably, $\sim 70 \%$ of the mice that received the islets initially in the eye retained their allografts throughout the follow-up after transplantation ( $>400$ days) (Fig. 1d) and only $50 \%$ of those transplanted in the kidney did with the same transient MR-1 treatment (Fig. 1e). The median survival times were 21 and 82.5 days in PBS- and Ig-treated control mice, respectively, when islets were transplanted in the eye, and 11 days when transplanted in the kidney of Ig-control mice. By contrast, $>50 \%$ of the mice treated with MR-1 retained their islet allografts in either site for $>300$ days after stopping the treatment. Moreover, mice exhibiting long-term survival of islet allografts (i.e. tolerant) were challenged with a second transplantation under the kidney capsule with the same peri-transplant MR-1 or Igcontrol treatments. The results showed that $\sim 30 \%$ of those initially transplanted in the kidney retained their second islet transplant for $\sim 400$ days after re-transplantation compared with $>70 \%$ of those initially transplanted in the eye (Fig. 1f).

Long-term survival of intraocular islet allografts in a baboon in the absence of immunosuppression We transplanted allogeneic (non-sibling) islets into the anterior chamber of the right eye of a diabetic recipient baboon $(n=1)$ that was treated transiently with anti-CD154 (CD40L) antibody (clone 5C8) in the peritransplantation period. The contralateral left eye did not receive any islets. Anti-CD154 antibody was administered intravenously at a dose of $20 \mathrm{mg} / \mathrm{kg}$ body weight on the day prior to transplant, the day of transplant and on POD 3, 10, 18, 28 and every 10 days thereafter until POD 248. We assessed the survival of the intraocular islet allografts before and after discontinuing anti-CD154 antibody treatment by direct non-invasive monitoring of the intraocular islet grafts as previously described [8]. These longitudinal eye examinations, lasting up to necropsy on POD 728, showed no change in the intraocular islet allografts during and after stopping immunosuppression (Fig. 2a) (i.e. 480 days of immunosuppression-free survival). Post-necropsy immunostaining of frozen sections of the eye bearing the islet grafts showed insulin- and glucagon-expressing cells within islets engrafted on top of the iris (Fig. 2b), further confirming survival and function of the islet allografts. Moreover, we assessed the graft survival and function during the longitudinal follow-up by measuring Cpeptide levels in the aqueous humour and plasma before and after stopping immunosuppression. C-peptide was considerably elevated in the eye bearing the islet grafts and was not detected in 

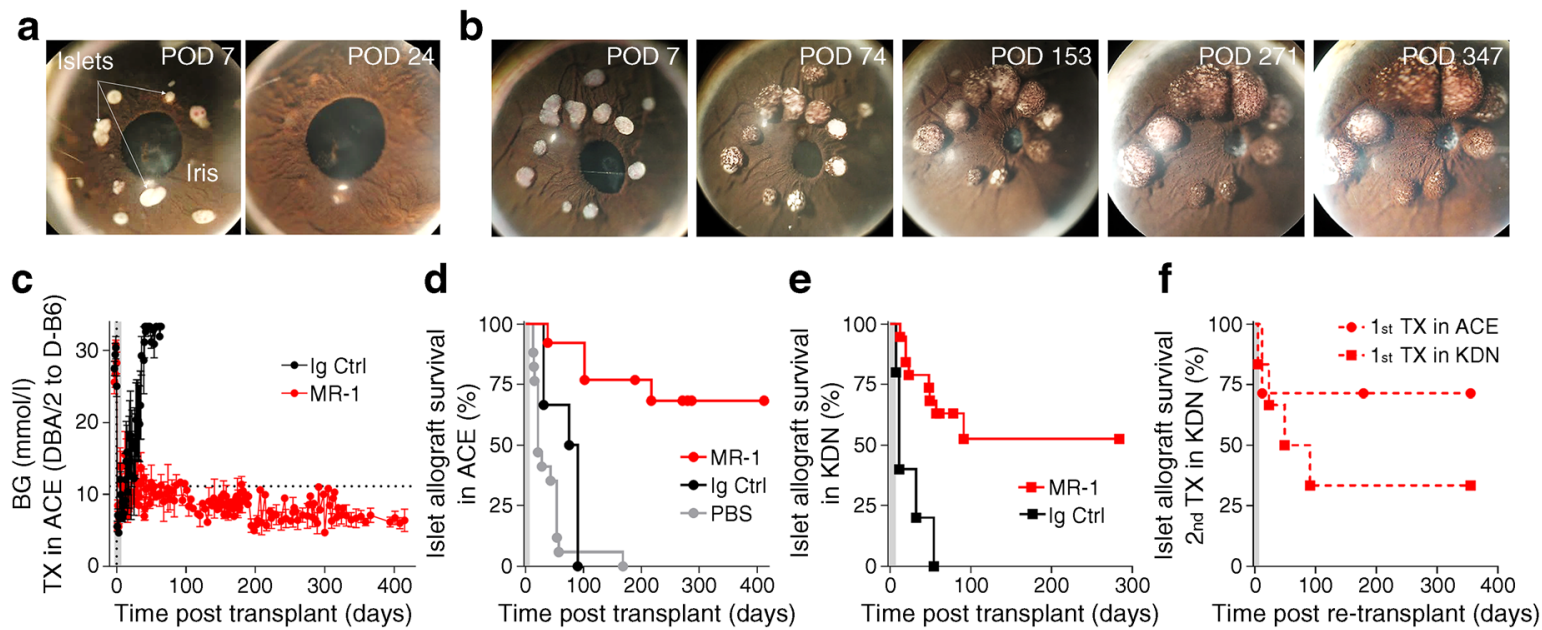

Fig. 1 Transient peri-transplantation anti-CD154 antibody treatment leads to long-term survival of intraocular islet allografts. (a, b) Representative longitudinal images of B6 mouse eyes transplanted with allogeneic DBA/2 islets in the anterior chamber of the eye while treated transiently with isotype Ig control/PBS (a) or anti-CD154 antibody (MR1) (b). Images on POD 7 show the transplanted islets engrafted on top of the iris that were rejected by POD 24 in mice treated with PBS/Ig control (a) but were still clearly visible on POD 347 in the anti-CD154-treated mice, long after stopping treatment on POD 7 (b). (c) Non-fasting blood glucose in STZ-induced diabetic B6 mice before and after transplantation of 250-300 IEQs (DBA/2) in the eye anterior chamber with MR-1 $(n=7)$ or Ig control $(n=5)$ treatments. Grey area indicates duration of the treatment. Normoglycaemia is defined as $<11.11 \mathrm{mmol} / 1$ (dotted horizontal line) and diabetes/hyperglycaemia as $>16.66 \mathrm{mmol} / 1$ (see also Methods).

the contralateral, non-transplanted eye (Fig. 2c; see also [8]). The median plasma C-peptide level was also increased compared with before transplantation, albeit not significantly (Fig. 2d). Repeated IVGTT before transplantation (POD -48) and after transplantation (POD 73, 128 and 204) showed increased plasma C-peptide during IVGTT only on POD 204 (Fig. 2e, f).

Cytokine profile in the intraocular islet allograft local environment in immune rejection vs tolerance Having the unique advantage of direct access to the intraocular islet allograft local in vivo environment, as represented by the aqueous humour, we measured cytokine levels in aqueous humour samples from the transplanted baboon and mice (Fig. 3). In mice, samples were collected from 'rejecting' recipients during ongoing acute destruction of the initial intraocular islet allografts (i.e. at rejection onset) and from mice that had either fully rejected ( $>20$ days post-rejection onset) or tolerated (tolerant; MR-1 treated) their islet allografts. Samples were also collected from nontransplanted control mice. The results showed that cytokine levels within the local environment of the islet grafts varied significantly between the conditions (Fig. 3a-f). Whereas the $\mathrm{T}$ helper (Th) 2 cytokines IL- 4 and TGF- $\beta 2$ were significantly decreased in rejecting mice (Fig. 3d, f), pro-inflammatory Th1/ Th17 cytokines such as IL-1 $\beta$, IFN- $\gamma$ and IL-17 $\alpha$ were significantly elevated compared with fully rejected or tolerant mice and with non-transplanted control mice (Fig. 3a-c). By contrast,

(d, e) Kaplan-Meier survival curves of islet allografts in diabetic B6 mice treated transiently (grey shaded areas) with MR-1/Ig control/PBS and transplanted initially (first transplant) either in the anterior chamber of the eye (d) or under the kidney capsule (e). For mice transplanted in the eye: MR-1 $n=13$, Ig control $n=6$ and PBS $n=17$; for mice transplanted in the kidney: MR-1 $n=19$ and Ig control $n=5$. (f) Survival of repeat transplant (second transplant) of islet allografts in the kidney following initial islet transplantation (first transplant) either in the anterior chamber of the eye or in the kidney. In MR-1-treated mice, median survival time was 70 days in mice initially transplanted in the kidney and remained undefined in those initially transplanted in the eye $(p=0.012$ by logrank Mantel-Cox test; also see ESM Fig. 1 for corresponding Ig controls). ACE, anterior chamber of the eye; BG, blood glucose; D-B6, diabetic B6; Ig Ctrl, Ig control; KDN, kidney; TX, transplant

TGF- $\beta 2$ was significantly elevated in tolerant compared with rejecting mice (Fig. 3f). IL-5 was also elevated in tolerant vs rejecting mice, albeit the difference did not reach significance (Fig. 3e). Notably, IL-4 was significantly elevated by more than fourfold in tolerant mice compared with the other conditions (Fig. 3d). A similar cytokine profile was observed in the baboon, where both IL-4 and IL-10 levels were increased in the graftbearing right eye on POD 429 compared with POD 31 and compared with the non-transplanted left eye (Fig. $3 g-j$ ).

Peripheral donor-specific immune regulation following intraocular islet transplantation We performed trans vivo DTH assays $[25,26]$ to assess whether local operational immune tolerance towards the intraocular islet allografts in the baboon precipitated peripheral immune regulation towards the donor. The results showed reduced DTH response with peripheral blood mononuclear cells (PBMCs) obtained from the recipient baboon (previously immunised for TT/D) upon repeat challenge with TT/D in the presence of soluble antigens from the islet donor (Fig. 4a, b). This was not observed with PBMCs from a nontransplanted, untreated control baboon that was also immunised for TT/D, even though the same soluble antigen preparation was co-injected (Fig. 4c). This linked suppression of the recall TT/D response in the recipient baboon was entirely donor-specific (Fig. 4d) and was abolished by blocking antibodies against IL-10, TGF- $\beta$ and IL-35 (IL-12 $\alpha[$ P35]/[Ebi3]] (Fig. 4e). 


\section{a}
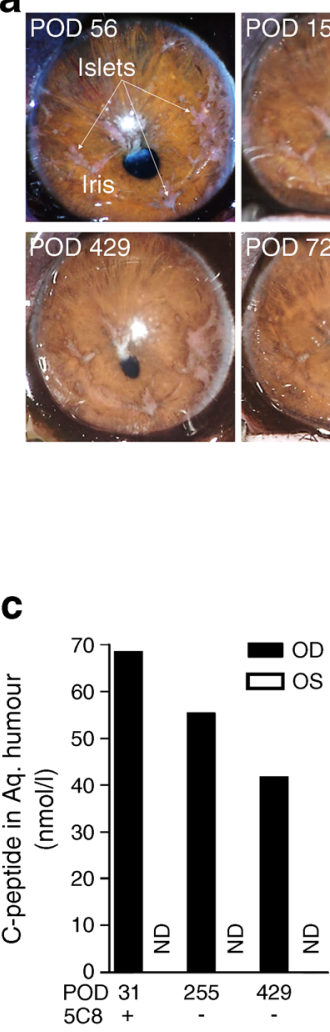

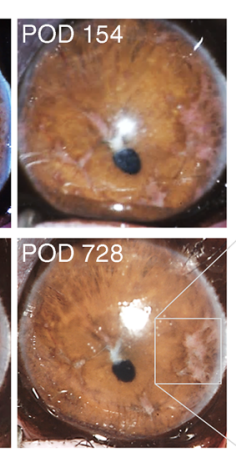

d

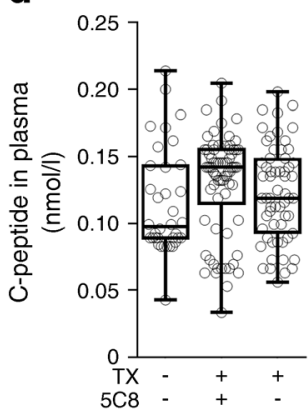

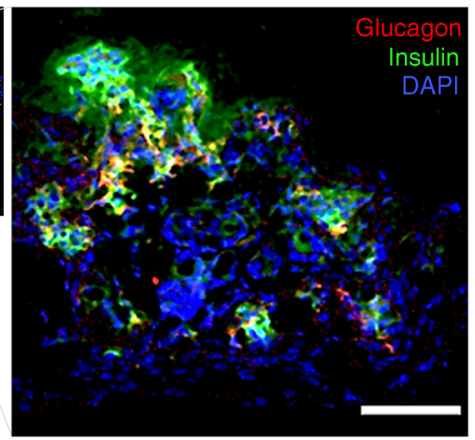

e $\rightarrow$ POD -48
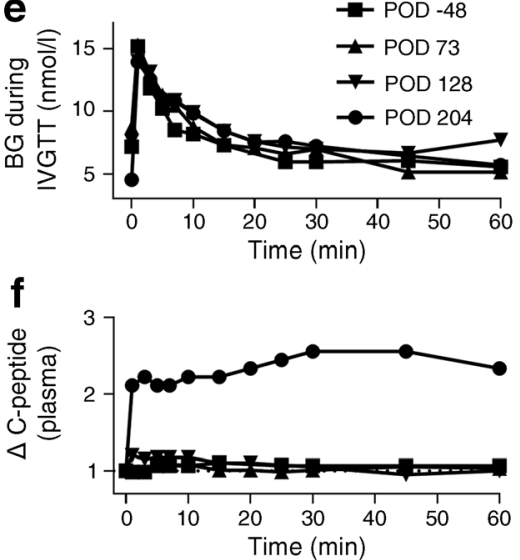

Fig. 2 Intraocular islet allografts survived and remained functional long after stopping anti-CD154 monotherapy in a diabetic baboon. (a) Longitudinal images of the baboon eye before (POD 56 and POD 154) and after (POD 429 and POD 728) stopping anti-CD154 (5C8) antibody treatment on POD 248. Inset shows intact islets on POD 728, which was 480 days after stopping immunosuppression. (b) Fluorescence micrographs showing positive insulin and glucagon immunostaining in a frozen eye section obtained after necropsy of the baboon on POD 728. (c) C-peptide levels in aqueous humour of the recipient baboon measured by electrochemiluminescence immunoassay. Aqueous humour samples were collected from the diabetic baboon islettransplanted right eye (OD) and non-transplanted left eye (OS) before (POD $31 ; n=1$ ) and after stopping anti-CD154 antibody treatment (POD

\section{Discussion}

We have previously shown the advantages of intraocular islet transplantation in studying non-invasively and longitudinally the immune responses mounted in vivo against allogeneic islets transplanted in the anterior chamber of the eye without immune intervention [13, 19]. We now present evidence of long-term survival of intraocular islet allografts consistent with operational graft immune tolerance, which was achieved with only transient immune intervention in the peri-transplant period. Animals treated with the anti-CD154 (CD40L) antibody retained their intraocular islet allografts for $>400$ days without immunosuppression. While mice transplanted in the kidney also showed prolonged survival of islet allografts with the same treatment, only $50 \%$ retained their grafts long-term compared with $70 \%$ of mice transplanted in the eye (Fig. 1a-e and ESM Fig. 1a). It should be noted that while the diabetic baboon still required insulin
255 and POD 429; $n=1$ each). (d) C-peptide levels in plasma of recipient baboon before/after islet transplantation and before/after stopping immunosuppression (5C8). The box and whisker plot shows the median values (horizontal black lines), the interquartile range, and the minimum and maximum values in each dataset (individual data points shown as white circles). (e) Blood glucose and (f) change in plasma C-peptide levels during $60 \mathrm{~min}$ IVGTTs performed before intraocular islet transplantation on POD -48 and after transplantation on POD 73, POD 128 and POD 204. C-peptide levels (shown as $\Delta$ C-peptide) were normalised to the mean (i.e. ratio) of values measured at -10 and -5 , and $0 \min (0 \mathrm{~min}=$ time of injection of glucose bolus; $0.5 \mathrm{~g} / \mathrm{kg}$ ) during the IVGTTs. Aq., aqueous; BG, blood glucose; ND, not detected; TX, transplant

therapy due to the small transplanted islet mass, its posttransplant plasma C-peptide levels were marginally increased compared with before transplantation ( $p=0.054$ by ANOVA) (Fig. 2d), likely due to the significant dilution of the aqueous humour C-peptide in the plasma as C-peptide levels changed in parallel in both compartments with fasting and post-feeding (see ESM Fig. 2 and ESM Table 1). A similar correlation was also observed in our previously studied diabetic baboon, which was transplanted with allogeneic islets in the eye but, in contrast to the currently studied baboon, was continuously maintained on immunosuppression [8]. Interestingly, while the daily insulin dose was modestly reduced post-transplant in the baboon in the current study (ESM Fig. 3a), the mean values of fasting and postprandial blood glucose levels were reduced significantly $(p<0.0001$ by ANOVA) following transplantation (ESM Fig. 3b-d). Thus, consistent with our previous observation [8], the current findings indicate that modest increases in circulating C-peptide originating 
Fig. 3 Cytokine profiles within the local islet environment varied significantly between rejection vs tolerance of intraocular islet allografts. (a-f) Cytokine levels measured by Bio-Plex assay in aqueous humour samples collected from B6 mice exhibiting long-term survival (tolerant; $n=$ 13 mice) or ongoing rejection (rejecting; $n=9$ ), or from mice that had completely rejected ( $>20$ days post rejection onset; $n=10)$ their intraocular islet allografts, as well as from non-transplanted B6 control mice (No TX Ctrl; $n=8$ ). Results are shown as means \pm SEM. $* p<0.05$ (by ANOVA). ( $\mathbf{g}-\mathbf{j})$ Cytokine levels measured by BioPlex assay in aqueous humour samples collected from the right (OD) and left (OS) eyes of the transplanted baboon during $5 \mathrm{C} 8$ treatment (POD 31; OD only; $n=$ 1 ) and after stopping $5 \mathrm{C} 8$ (antiCD154) treatment on POD 429 ( $n=1$ each). ND, not detected; $\mathrm{TX}$, transplant
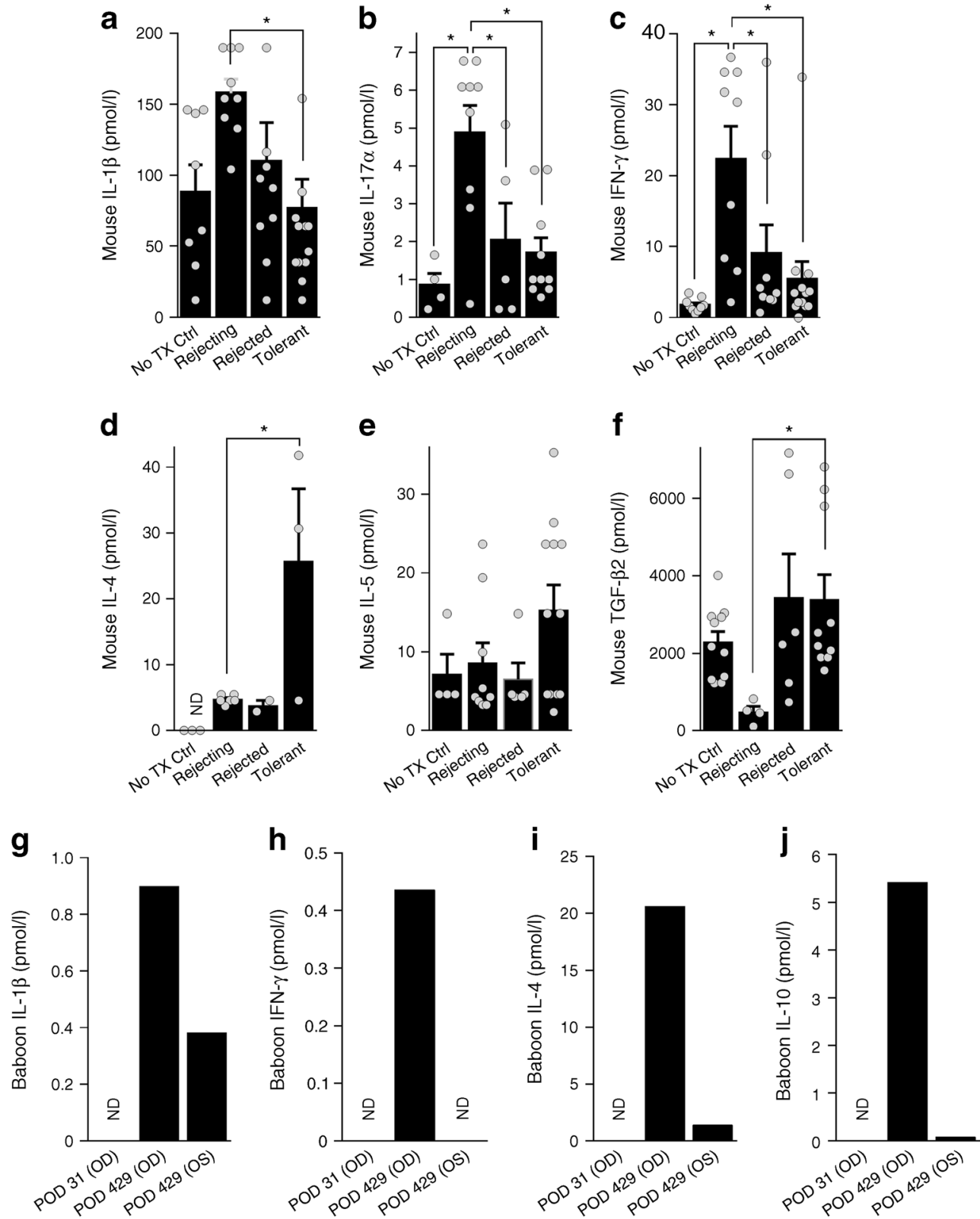

from even a small mass of intraocular islet grafts can result in improvement in overall blood glucose control in this clinically relevant non-human primate model [9]. This is in line with clinical data from individuals with type 1 diabetes showing significant improvement in quality of life through better glycaemic control following intrahepatic islet transplantation, and even after resumption of insulin therapy due to graft failure [1, 27].

Intraocular islet transplantation has technical advantages that can be uniquely beneficial in preclinical and clinical applications $[8,9,13,28,29]$. Although long-term survival of islet allografts has been achieved using various immune conditioning protocols in preclinical models of islet transplantation to other sites [30-33], including (by us) in the kidney subcapsular space (Fig. 1e and ESM Fig. 1a), intraocular islet transplantation allows quantitative monitoring of the same individual islets non- invasively and longitudinally. An advantage of this technique is that it revealed an unexpected increase over time in the size/volume of the surviving islet allografts in the tolerant mice (Fig. 1b). While unravelling the molecular mechanisms underlying this impressive islet growth is still needed and requires dedicated studies beyond the scope of the current study, we speculate that the augmented metabolic demand on the marginal mass of the intraocular islet graft, consequent to increased body mass of the recipients over time, may be involved (see ESM Fig. 4) [34, 35]. Additionally, intraocular islet transplantation uniquely allows access to the local graft environment in vivo. We have taken advantage of this to gain insight into the immune mechanisms underlying the intraocular islet allograft's long-term survival. The results revealed significantly elevated local levels of IL-4 among other immune regulatory cytokines (e.g. IL-10) in both our 

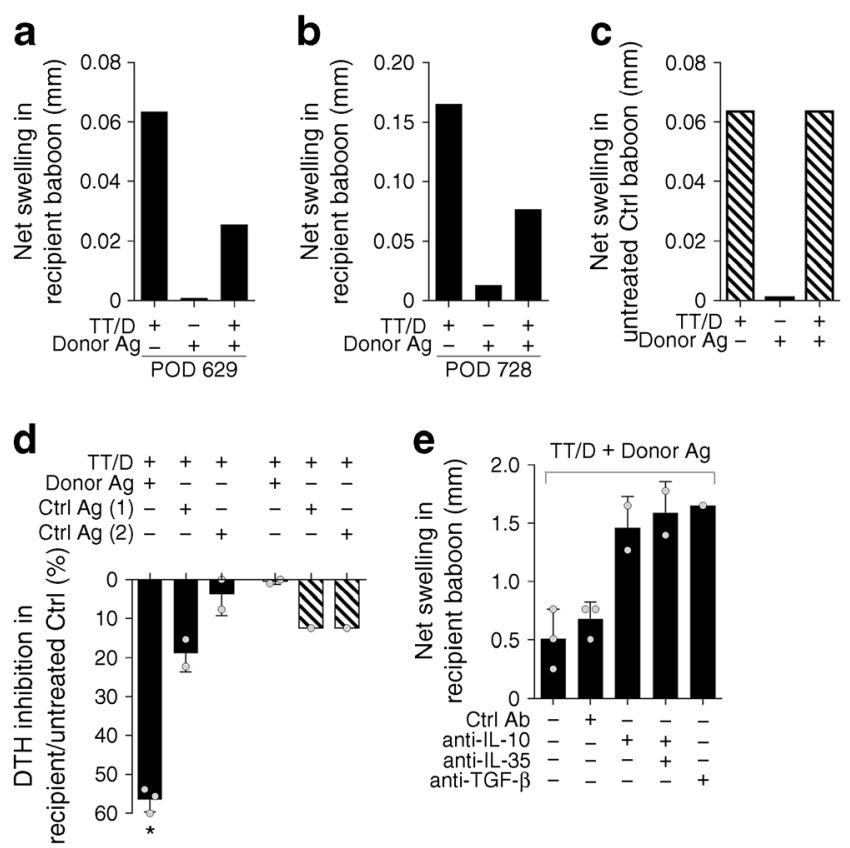

Fig. 4 Operational immune tolerance of intraocular islet allografts associated with donor-specific peripheral immune regulation (bystander suppression) in trans vivo DTH assay. (a-c) Net swelling in trans vivo DTH by the recipient baboon on POD 629 (a) and POD 728 (b) and an untreated control (Ctrl) baboon (non-transplanted) (c) in response to challenge by recall antigen TT/D alone (positive control), donor antigen (Donor Ag) and a mixture of both ( $n=1$ in each condition). (d) Per cent inhibition of the recall response to TT/D by the recipient baboon (black bars) and nontransplanted untreated control (Ctrl) baboon (hatched bars) in the presence of soluble antigens from the specific donor baboon from which islets were isolated (Donor Ag) and naive third-party control baboons ( $\mathrm{Ctrl} \mathrm{Ag} \mathrm{[1]} \mathrm{and}$ Ctrl Ag [2]; $n=1$ each). Swelling data in the different conditions were normalised to response to TT/D alone and pooled from repeat trans vivo DTH assays ( $n=3$ for recipient baboon on POD 629, POD 665 and POD $728 ; n=2$ for untreated control) and presented as means \pm SD (see also Methods). $* p<0.05$ (by unpaired Student's $t$ test) vs control antigens. (e) Cytokine dependence of donor-specific linked immune regulation in the recipient baboon. DTH recall response (shown as net swelling) to TT/D by the recipient baboon in the presence of the donor baboon antigens (Donor $\mathrm{Ag}$ ) without and with blocking antibodies against IL-10, IL-35 (anti-IL$12[\mathrm{P} 35]+$ anti-Ebi3) and TGF- $\beta$, or Ig isotype control $(\mathrm{Ctrl} \mathrm{Ab})$. Data shown as means $\pm \mathrm{SD}$

mouse and non-human primate models (Fig. 3). IL-4 signalling through the IL-4R $\alpha$ /STAT6 pathway has been shown to promote Th2 cytokine production and in turn the polarisation of innate/ adaptive immune cells towards regulatory function [36, 37]. IL-4 is also implicated in cancer-associated immune regulation within the tumour microenvironment to avoid immune clearance [38]. Consistently, our current findings suggest the significant local role of IL-4 among other Th2 cytokines and associated local immune regulatory mechanisms in the observed operational immune tolerance towards intraocular islet allografts.

Remarkably, assessment of immune reactivity in the periphery of the recipient baboon following intraocular islet transplant showed in trans vivo DTH assay donor-specific immune regulation long after stopping immunosuppression (Fig. 4). DTH is a peripheral immune response by antigen-experienced $\mathrm{T}$ cells that occurs rapidly in vivo upon repeat exposure to, or challenge with, the specific antigen(s). Hence, a DTH reaction requires initial host sensitisation to the specific antigen(s) and, therefore, lack of a DTH response to recall antigen(s) is evidence for either antigen-specific peripheral immune hyporesponsiveness (or anergy) or active immune regulation/suppression of an effector immune response by antigen-specific regulatory cells. Both anergy and immune regulation are important components of peripheral immune tolerance [39-41]. While the DTH response has been reliably used clinically to assess prior exposure to infectious or immunising agents, its utility in transplant recipients to assess immune reactivity (or lack thereof) to the graft donor is limited due to the risk of sensitising the recipient to donor antigens and consequent triggering of graft rejection or loss. To circumvent this limitation, the trans vivo DTH assay was developed wherein immune reactivity of the transplant recipient towards the donor is assessed outside the recipient in live mice [25], and other in vitro assays have been used, such as mixed leucocyte reaction (MLR), measuring donor-specific antibody titres, and tetramer and elispot analyses [42, 43]. However, all these methods have some shortcomings. In the trans vivo DTH assay, PBMCs are obtained from the recipient and injected into the footpad of a mouse where the DTH-type response to a known, previously exposed-to antigen(s) through natural exposure or vaccination (e.g. TT/D), is measured based on local swelling [26]. The swelling occurs in the highly vascular mouse tissue because of local inflammation consequent to exposure and activation of the transplant recipient's antigenexperienced $\mathrm{T}$ cells to the corresponding donor antigens; this immune reaction also attracts mouse immune cells resulting in further local inflammation manifesting in oedema and swelling of the footpad. This inflammatory immune response is consistent with a positive in vivo skin recall DTH response in humans. Alternatively, reduced swelling (i.e. DTH response) is indicative of antigen-specific hyporesponsiveness, likely due to bystander immune suppression by regulatory cells among the injected PBMCs of the transplant recipient. In our studies (Fig. 4), PBMCs were obtained from the islet donor, thirdparty control baboons, the recipient baboon and a nontransplanted untreated control baboon. The results showed a $60 \%$ inhibition in the DTH response by the recipient only in the presence of antigens of the specific islet donor. Interestingly, this in vivo immune regulation/tolerance was dependent on IL10 , TGF- $\beta$ and IL-35, thereby suggesting a prominent involvement of various subsets of T regulatory cells (Treg) in the observed peripheral immune hyporesponsiveness by the recipient towards the specific donor. A similar pattern of peripheral immune regulation has previously been described in humans and Rhesus monkey due to tolerance to non-inherited maternal antigens [44], as well as in B6 mice made tolerant by donorspecific transfusion plus costimulation blockade [44, 45]. Importantly, while the recipient baboon's recall response to TT/D was significantly reduced in the presence of the islet 
donor's antigens, its response in the presence of third-party antigens was equal to that by the untreated control baboon, thus confirming re-established immune competence of the recipient after stopping immunosuppression (see ESM Fig. 5). Together, these results obtained in one diabetic baboon are consistent with donor-specific peripheral immune tolerance in the recipient and emphasise the importance of further corroborating these findings in a larger number of non-human primates.

We investigated this notion further in mice exhibiting immune tolerance towards allogeneic islets transplanted initially either in the eye or in the kidney by challenging them with repeat transplantation with islets from the same donors in the periphery (i.e. kidney). Interestingly, $\sim 72 \%$ of the MR-1treated mice initially transplanted in the eye retained their second islet allograft in the kidney (repeat transplant) compared with $\sim 33 \%$ of those initially transplanted in the kidney (Fig. 1f and ESM Fig. 1b). While additional studies are needed to further elaborate on the mechanisms underlying the induction and maintenance of the observed operational immune tolerance towards the islet allografts in the eye and periphery, the current findings point to a distinct advantage of using the eye over the kidney upon follow-up transplantation. This is conceptually significant and potentially has broad implications in transplant applications, where a priori donor/tissuespecific immune tolerance is established through the intraocular route in conjunction with transient immune interventions and followed later by transplantation in the periphery of additional tissues/cells from the same donor/source (e.g. stem-cell derived). Although it remains to be examined clinically, this staggered approach could address the potential eye limitation in accommodating sufficient islet mass to achieve insulin independence in individuals with type 1 diabetes [9].

In summary, our current studies provide proof-of-concept evidence for operational immune tolerance towards allogeneic pancreatic islets transplanted into the anterior chamber of the eye, with a higher potential for associated donor-specific immune tolerisation in the periphery when compared with the kidney. This was achieved only when transient peri-transplant immune intervention was implemented. It should be emphasised, however, that while our current findings are significant for potential clinical application, additional studies (particularly with non-human primates) are needed to establish this new approach to inducing immune tolerance in islet transplantation through the intraocular route in conjunction with transient peri-transplant immune intervention. Moreover, our current studies were conducted using immune costimulatory blockade with anti-CD154 antibody clones that are different from the earlier humanised clone that caused thromboembolic complications in initial clinical trials [46, 47]. Although the mechanism for such complications has been resolved and new humanised clones have been developed [48, 49], our approach must be evaluated using the new clone(s) or other clinically relevant immune interventions. Additional studies, preferably with non-human primates, will also be needed to establish the therapeutic mass of islets transplanted in the eye and to develop new transient immune regimens that would be effective with and without a background of autoimmune type 1 diabetes. These features of intraocular islet transplantation combined with the above-described and previously demonstrated technical advantages underscore its potential impact in clinical application. Coming on the heels of a phase 1 clinical trial on intraocular islet transplantation in legally blind patients with type 1 diabetes (ClinicalTrials.gov registration no. NCT02846571), the current findings may have significant implications in islet transplantation to treat type 1 diabetes sooner than anticipated.

Acknowledgements The authors are grateful to W. Diaz, J. Geary and R. Rodriguez-Lopez (Diabetes Research Institute, University of Miami, USA) for their excellent care of non-human primates and associated procedures, and to A. Rabassa and E. Poumian-Ruiz (Diabetes Research Institute, University of Miami, USA) for assistance with islet isolation from the donor baboon. We also thank S. Dubovy and C. Maza (Bascom Palmer Eye Institute, University of Miami, USA) for help with sectioning and histological examination of the baboon eyes, and A. Mendez (Diabetes Research Institute, University of Miami, USA) and H. SalahUddin (Department of Psychiatry, University of Miami, USA) for help with Bio-Plex assay setup.

Data availability Data supporting the results reported in this article are available on request from the authors.

Funding This work was supported by funds from the Diabetes Research Institute Foundation (DRIF) and the Diabetes Wellness Foundation and by grants from the Stanley J. Glaser Foundation Research Award (UM SJG2016-2), the NIH/NIDDK/NIAID (K01DK097194, U01-AI-102456, R56AI130330, UC4DK116241), the Swedish Diabetes Association Fund, the Swedish Research Council, Novo Nordisk Foundation, the Family Erling-Persson Foundation, Strategic Research Program in Diabetes at Karolinska Institutet, the ERC-2013-AdG 338936BetaImage, the Family Knut and Alice Wallenberg Foundation, Skandia Insurance Company Ltd, Diabetes and Wellness Foundation, the Bert von Kantzow Foundation and the Stichting af Jochnick Foundation.

Duality of interest P-OB is cofounder and CEO of Biocrine, an unlisted biotech company that is using the anterior chamber of the eye technique as a research tool. MHA is consultant for the same company. All other authors declare that there is no duality of interest associated with their contribution to this manuscript.

Contribution statement MHA conceived the study, designed and conducted experiments, analysed and interpreted data and wrote the manuscript. DMB designed and conducted experiments, analysed and interpreted data and wrote the manuscript. AS, CM, MH, AT, LFH, AH and EAA-Q conducted experiments and collected data and proofread the manuscript. JMP planned experiments, interpreted data and proofread the manuscript. WJB and EJ-G performed trans vivo DTH assays, interpreted data and edited the manuscript. VLP designed experiments, performed intraocular islet transplantation and eye examinations in the baboon, interpreted data and edited the manuscript. CR, NSK and P-OB conceived the study, designed experiments, interpreted data and edited the manuscript. All authors approved the version of the manuscript to be published. MHA, DMB, and $\mathrm{P}-\mathrm{OB}$ are the guarantors of this work.

Publisher's note Springer Nature remains neutral with regard to jurisdictional claims in published maps and institutional affiliations. 


\section{References}

1. Hering BJ, Clarke WR, Bridges ND et al (2016) Phase 3 trial of transplantation of human islets in type 1 diabetes complicated by severe hypoglycemia. Diabetes Care 39(7):1230-1240. https://doi. org/10.2337/dc15-1988

2. Tharavanij T, Betancourt A, Messinger S et al (2008) Improved long-term health-related quality of life after islet transplantation. Transplantation 86(9):1161-1167. https://doi.org/10.1097/TP. 0b013e31818a7f45

3. Shapiro AM (2012) Islet transplantation in type 1 diabetes: ongoing challenges, refined procedures, and long-term outcome. Rev Diabet Stud 9(4):385-406. https://doi.org/10.1900/RDS.2012.9.385

4. Berman DM, Molano RD, Fotino C et al (2016) Bioengineering the endocrine pancreas: intraomental islet transplantation within a biologic resorbable scaffold. Diabetes 65(5):1350-1361. https://doi. org $10.2337 / \mathrm{db} 15-1525$

5. Wolf-van Buerck L, Schuster M, Baehr A et al (2015) Engraftment and reversal of diabetes after intramuscular transplantation of neonatal porcine islet-like clusters. Xenotransplantation 22(6):443450. https://doi.org/10.1111/xen.12201

6. Maffi P, Balzano G, Ponzoni M et al (2013) Autologous pancreatic islet transplantation in human bone marrow. Diabetes 62(10):35233531. https://doi.org/10.2337/db13-0465

7. Korsgren O, Nilsson B (2009) Improving islet transplantation: a road map for a widespread application for the cure of persons with type I diabetes. Curr Opin Organ Transplant 14(6):683-687. https:// doi.org/10.1097/MOT.0b013e328332c44c

8. Perez VL, Caicedo A, Berman DM et al (2011) The anterior chamber of the eye as a clinical transplantation site for the treatment of diabetes: a study in a baboon model of diabetes. Diabetologia 54(5): 1121-1126. https://doi.org/10.1007/s00125-011-2091-y

9. Shishido ACA, Rodriguez-Diaz R, Berggren P-O, Abdulreda MH (2016) Clinical intraocular islet transplantation is not a number issue. CellR4 4:e2120

10. Szot GL, Yadav M, Lang J et al (2015) Tolerance induction and reversal of diabetes in mice transplanted with human embryonic stem cell-derived pancreatic endoderm. Cell Stem Cell 16(2):148157. https://doi.org/10.1016/j.stem.2014.12.001

11. Lee K, Nguyen V, Lee KM, Kang SM, Tang Q (2014) Attenuation of donor-reactive $\mathrm{T}$ cells allows effective control of allograft rejection using regulatory $\mathrm{T}$ cell therapy. Am J Transplant 14(1):27-38. https://doi.org/10.1111/ajt.12509

12. Speier $\mathrm{S}$, Nyqvist $\mathrm{D}$, Cabrera $\mathrm{O}$ et al (2008) Noninvasive in vivo imaging of pancreatic islet cell biology. Nat Med 14(5):574-578. https://doi.org/10.1038/nm1701

13. Abdulreda MH, Faleo G, Molano RD et al (2011) High-resolution, noninvasive longitudinal live imaging of immune responses. Proc Natl Acad Sci U S A 108(31):12863-12868. https://doi.org/10. 1073/pnas. 1105002108

14. Abdulreda MH, Rodriguez-Diaz R, Caicedo A, Berggren PO (2016) Liraglutide compromises pancreatic beta cell function in a humanized mouse model. Cell Metab 23(3):541-546. https://doi. org/10.1016/j.cmet.2016.01.009

15. Carlsson PO, Palm F (2002) Oxygen tension in isolated transplanted rat islets and in islets of rat whole-pancreas transplants. Transpl Int 15(11):581-585. https://doi.org/10.1111/j.1432-2277. 2002.tb00112.x

16. McLaren JW, Dinslage S, Dillon JP, Roberts JE, Brubaker RF (1998) Measuring oxygen tension in the anterior chamber of rabbits. Invest Ophthalmol Vis Sci 39(10):1899-1909

17. Sharifipour F, Yazdani S, Pakravan M, Idani E (2013) Aqueous oxygen tension in glaucomatous and nonglaucomatous eyes. J Glaucoma 22(8):608-613. https://doi.org/10.1097/IJG. 0b013e318255bc62
18. Nyqvist D, Speier S, Rodriguez-Diaz R et al (2011) Donor islet endothelial cells in pancreatic islet revascularization. Diabetes 60(10):2571-2577. https://doi.org/10.2337/db10-1711

19. Miska J, Abdulreda MH, Devarajan P et al (2014) Real-time immune cell interactions in target tissue during autoimmune-induced damage and graft tolerance. J Exp Med 211(3):441-456. https://doi. org/10.1084/jem.20130785

20. Berman DM, Cabrera O, Kenyon NM et al (2007) Interference with tissue factor prolongs intrahepatic islet allograft survival in a nonhuman primate marginal mass model. Transplantation 84(3):308315. https://doi.org/10.1097/01.tp.0000275401.80187.1e

21. Kenyon NS, Fernandez LA, Lehmann R et al (1999) Long-term survival and function of intrahepatic islet allografts in baboons treated with humanized anti-CD154. Diabetes 48(7):1473-1481. https://doi.org/10.2337/diabetes.48.7.1473

22. Pileggi A, Molano RD, Berney T et al (2001) Heme oxygenase-1 induction in islet cells results in protection from apoptosis and improved in vivo function after transplantation. Diabetes 50(9):19831991. https://doi.org/10.2337/diabetes.50.9.1983

23. Abdulreda MH, Caicedo A, Berggren P-O (2013) Transplantation into the anterior chamber of the eye for longitudinal, non-invasive in vivo imaging with single-cell resolution in real-time. J Vis Exp: e50466

24. Han D, Berman DM, Willman M et al (2010) Choice of immunosuppression influences cytomegalovirus DNAemia in cynomolgus monkey (Macaca fascicularis) islet allograft recipients. Cell Transplant 19(12):1547-1561. https://doi.org/10.3727/ 096368910X513973

25. Burlingham WJ, Jankowska-Gan E, VanBuskirk A, Orosz CG, Lee JH, Kusaka S (2000) Loss of tolerance to a maternal kidney transplant is selective for HLA class II: evidence from trans-vivo DTH and alloantibody analysis. Hum Immunol 61(12):1395-1402. https://doi.org/10.1016/S0198-8859(00)00217-2

26. Jankowska-Gan E, Hegde S, Burlingham WJ (2013) Trans-vivo delayed type hypersensitivity assay for antigen specific regulation. J Vis Exp:e4454

27. Cure P, Pileggi A, Froud T et al (2008) Improved metabolic control and quality of life in seven patients with type 1 diabetes following islet after kidney transplantation. Transplantation 85(6):801-812. https://doi.org/10.1097/TP.0b013e318166a27b

28. Abdulreda MH, Berggren PO (2013) Islet inflammation in plain sight. Diabetes Obes Metab 15(Suppl 3):105-116. https://doi.org/ 10.1111/dom. 12160

29. Rodriguez-Diaz R, Speier S, Molano RD et al (2012) Noninvasive in vivo model demonstrating the effects of autonomic innervation on pancreatic islet function. Proc Natl Acad Sci U S A 109(52): 21456-21461. https://doi.org/10.1073/pnas.1211659110

30. Faustman DL, Steinman RM, Gebel HM, Hauptfeld V, Davie JM, Lacy PE (1984) Prevention of rejection of murine islet allografts by pretreatment with anti-dendritic cell antibody. Proc Natl Acad Sci U S A 81(12):3864-3868. https://doi.org/10.1073/pnas.81.12.3864

31. Terasaka R, Lacy PE, Hauptfeld V, Bucy RP, Davie JM (1986) The effect of cyclosporin-A, low-temperature culture, and anti-Ia antibodies on prevention of rejection of rat islet allografts. Diabetes 35(1):83-88. https://doi.org/10.2337/diab.35.1.83

32. Giovannoni L, Muller YD, Lacotte S et al (2015) Enhancement of islet engraftment and achievement of long-term islet allograft survival by toll-like receptor 4 blockade. Transplantation 99(1):29-35. https://doi.org/10.1097/TP.0000000000000468

33. Koulmanda M, Qipo A, Fan Z et al (2012) Prolonged survival of allogeneic islets in cynomolgus monkeys after short-term triple therapy. Am J Transplant 12(5):1296-1302. https://doi.org/10. 1111/j.1600-6143.2012.03973.x

34. Almaca J, Molina J, Arrojo EDR et al (2014) Young capillary vessels rejuvenate aged pancreatic islets. Proc Natl Acad Sci U S A 111(49):17612-17617. https://doi.org/10.1073/pnas.1414053111 
35. Chmelova H, Cohrs CM, Chouinard JA et al (2015) Distinct roles of $\beta$-cell mass and function during type 1 diabetes onset and remission. Diabetes 64(6):2148-2160. https://doi.org/10.2337/db14-1055

36. Ul-Haq Z, Naz S, Mesaik MA (2016) Interleukin-4 receptor signaling and its binding mechanism: a therapeutic insight from inhibitors tool box. Cytokine Growth Factor Rev 32:3-15. https://doi.org/10. 1016/j.cytogfr.2016.04.002

37. Paul WE (2015) History of interleukin-4. Cytokine 75(1):3-7. https://doi.org/10.1016/j.cyto.2015.01.038

38. Roth F, De La Fuente AC, Vella JL, Zoso A, Inverardi L, Serafini P (2012) Aptamer-mediated blockade of IL $4 \mathrm{R} \alpha$ triggers apoptosis of MDSCs and limits tumor progression. Cancer Res 72(6):13731383. https://doi.org/10.1158/0008-5472.CAN-11-2772

39. Xu Q, Lee J, Jankowska-Gan E et al (2007) Human CD $4^{+} \mathrm{CD} 25^{\text {low }}$ adaptive $\mathrm{T}$ regulatory cells suppress delayed-type hypersensitivity during transplant tolerance. J Immunol 178(6):3983-3995. https:// doi.org/10.4049/jimmunol.178.6.3983

40. Torrealba JR, Katayama M, Fechner JH Jr et al (2004) Metastable tolerance to rhesus monkey renal transplants is correlated with allograft TGF- $\beta 1^{+} \mathrm{CD} 4^{+} \mathrm{T}$ regulatory cell infiltrates. J Immunol 172(9):5753-5764. https://doi.org/10.4049/jimmunol.172.9.5753

41. Billingham RE, Brent L, Medawar PB (1953) Actively acquired tolerance of foreign cells. Nature 172(4379):603-606. https://doi. org/10.1038/172603a0

42. Gonzalez-Nieto L, Domingues A, Ricciardi M et al (2016) Analysis of simian immunodeficiency virus-specific CD8+ T cells in Rhesus macaques by peptide-MHC-I tetramer staining. J Vis Exp:e54881
43. Nadazdin O, Boskovic S, Murakami T et al (2011) Host alloreactive memory $\mathrm{T}$ cells influence tolerance to kidney allografts in nonhuman primates. Sci Transl Med 3:86ra51

44. Tomita Y, Satomi M, Bracamonte-Baran W et al (2016) Kinetics of alloantigen-specific regulatory CD4 T cell development and tissue distribution after donor-specific transfusion and costimulatory blockade. Transplant Direct 2(5):e73. https://doi.org/10.1097/ TXD.0000000000000580

45. Burlingham WJ, Jankowska-Gan E, Kempton S, Haynes L, Kaufman DB (2015) Patterns of immune regulation in rhesus macaque and human families. Transplant Direct 1:e20

46. Kawai T, Andrews D, Colvin RB, Sachs DH, Cosimi AB (2000) Thromboembolic complications after treatment with monoclonal antibody against CD40 ligand. Nat Med 6(2):114. https://doi.org/ $10.1038 / 72162$

47. Boumpas DT, Furie R, Manzi S et al (2003) A short course of BG9588 (anti-CD40 ligand antibody) improves serologic activity and decreases hematuria in patients with proliferative lupus glomerulonephritis. Arthritis Rheum 48(3):719-727. https://doi.org/10. 1002/art.10856

48. Pinelli DF, Ford ML (2015) Novel insights into anti-CD40/CD154 immunotherapy in transplant tolerance. Immunotherapy 7(4):399 410. https://doi.org/10.2217/imt.15.1

49. Kim SC, Wakwe W, Higginbotham LB et al (2017) Fc-silent antiCD154 domain antibody effectively prevents nonhuman primate renal allograft rejection. Am J Transplant 17(5):1182-1192. https://doi.org/10.1111/ajt.14197 